\title{
Modelling and accuracy estimation of a new omnidirectional depth computation sensor
}

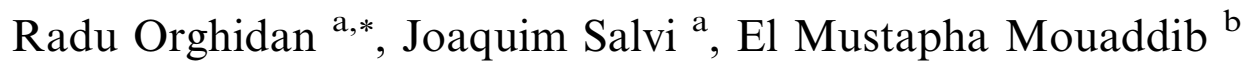 \\ ${ }^{a}$ Institute of Informatics and Applications, Computer Vision and Robotics Group, University of Girona, Edifici P-IV, \\ Campus Montilivi, 17071 Girona, Spain \\ ${ }^{\mathrm{b}}$ Centre of Robotics, Electrotechnics and Automation, University of Picardie Jules Verne, Amiens, France \\ Received 23 May 2005; received in revised form 30 September 2005 \\ Available online 21 February 2006 \\ Communicated by R. Davies
}

\begin{abstract}
Depth computation is an attractive feature in computer vision. The use of traditional perspective cameras for panoramic perception requires several images, most likely implying the use of several cameras or of a sensor with mobile elements. Moreover, misalignments can appear for non-static scenes. Omnidirectional cameras offer a much wider field of view (FOV) than perspective cameras, capture a panoramic image at every moment and alleviate problems due to occlusions. A practical way to obtain depth in computer vision is the use of structured light systems. This paper is focused on combining omnidirectional vision and structured light with the aim of obtaining panoramic depth information. The resulting sensor is formed by a single catadioptric camera and an omnidirectional light projector. The model and the prototype of a new omnidirectional depth computation sensor are presented in this article and its accuracy is estimated by means of laboratory experimental setups.
\end{abstract}

(C) 2005 Elsevier B.V. All rights reserved.

Keywords: Omnidirectional vision; Catadioptrics; Calibration; Structured light; 3D reconstruction

\section{Introduction}

Vision is certainly the most important of the five senses. It is a complicated process that requires numerous components of the human eye and brain to work together. However, binocular human vision has a limited field of view

\footnotetext{
This work is partially supported by the Spanish project CICYT TIC 2003-08106-C02-02 and by the AIRE mobility grant provided by the Government of Catalunya allowing a four month stay for Radu Orghidan at the University of Picardie.

* Corresponding author. Tel.: +34 639 612515; fax: +34 972418098.

E-mail addresses: radu@eia.udg.es, rorghi@yahoo.com (R. Orghidan), qsalvi@eia.udg.es (J. Salvi), mouaddib@u-picardie.fr (E.M. Mouaddib).

URLs: http://eia.udg.es/ radu/ (R. Orghidan), http://eia.udg.es/ $\sim$ qsalvi/ (J. Salvi), http://www.crea.u-picardie.fr/ mouaddib/ (E.M. Mouaddib).
}

(Yagi, 1999) which is complemented by peripheral vision. In specific real-life situations, such as vehicle driving, a even wider vision field is required and vision is enhanced by the use of reflecting surfaces. The main problem of this kind of systems is the dead angles, the regions in which objects cannot be seen due to the physical limitations of the mirrors. This problem can be overcome using omnidirectional vision sensors which ideally have the capability to see in all directions (360) simultaneously.

\subsection{Omnidirectional vision sensors}

Besides these obvious advantages offered by a large field of view, in robot navigation the necessity of employing omnidirectional sensors also stems from a well known problem in computer vision: the motion estimation algorithms can sometimes mistake a small pure translation of 
the camera for a small rotation, and the possibility of error increases if the field of view is narrow or the depth variations in the scene are small. An omnidirectional sensor can reduce this error since it receives more information for the same movement of the camera than the one obtained by a reduced field of view. A common classification of existing omnidirectional sensors is related to the components used for their fabrication. Thus, the field of view of conventional cameras is enhanced by: (a) fish-eye lenses; (b) multiple image acquisition systems using rotating cameras or structures of many cameras with complementary fields of view; (c) cameras in front of especial mirrors (planar, hyperbolic, parabolic, spherical, dual convex and conic). Yagi (1999) surveyed the existing techniques to build cameras with a wide field of view and Svoboda and Pajdla (2000) proposed several classifications of the existing omnidirectional cameras according to their most important features.

Beginning in the early nineties, the attention given to omnidirectional vision and its knowledge base has grown continuously as the numbers of researchers involved in the study of omnidirectional cameras has increased. New mathematical models for catadioptric projection and, consequently, better performing catadioptric sensors have appeared. The catadioptric sensors use reflecting surfaces (convex or planar mirrors) coupled to a conventional camera. In the commercial applications, the large interest generated by catadioptrics is due to their specific advantages when compared to other omnidirectional systems, especially the price and the compactness. In 1990, the Japanese team from Mitsubishi Electric Corporation, lead by Yagi, studied the panoramic scenes generated using a conic mirror-based sensor (Yagi and Kawato, 1990). The sensor, named COPIS, was used for generating the environmental map of an indoor scene from a mobile robot. The conic mirror shape was also used, in 1995, by the researchers from CREA, University of Picardie Jules Verne. Their robot was provided with an omnidirectional sensor, bap- tized SYCLOP, and was used for navigation and localization in 3D space (Pegard and Mouaddib, 1996).

The catadioptric sensors can be classified depending on the way they gather light rays. When all the observed light rays converge into a point, called the focus, the sensors are known as Single View Point (SVP). In 1996 Bruckstein and Richardson (2000) compared several mirror shapes and thoughtfully studied the parabolic and the hyperbolic profiles, the most frequent used mirrors in SVP sensors. Later, Baker and Nayar (1998) explored the catadioptric sensors that produce SVP. Using geometrical relations, they found the equation of the single view point constraint. The solutions for this equation represent the entire class of mirrors that respect the constraint. While some of these solutions are impractical, others define the following set of feasible mirrors: planar, conical, spherical, ellipsoidal, hyperboloid and parabolic. In the case of the hyperbolic mirrors, the SVP constraint is satisfied when the pinhole and the mirror view point are placed at the two foci of the hyperboloid. The parabolic shape is a solution for the SVP constraint when the image is formed by an orthographic projection, see Fig. 1.

The catadioptric sensors that do not possess a single view point (non-SVP) are less used but have proved to be helpful for applications with specific requirements such as prescribed distortions (Hicks and Perline, 2001) or with linear projection constraints (Gaspar et al., 2002).

\subsection{Previous work in panoramic $3 D$ modelling with catadioptric sensors}

Our current research is focused on modelling 3D real scenes using catadioptric sensors. As shown in the previous section, there are many ways to obtain wide field of view sensors but neither the standard cameras nor the catadioptric ones can provide depth information about the scene when used independently. A method for obtaining 3D information using a single catadioptric camera performing

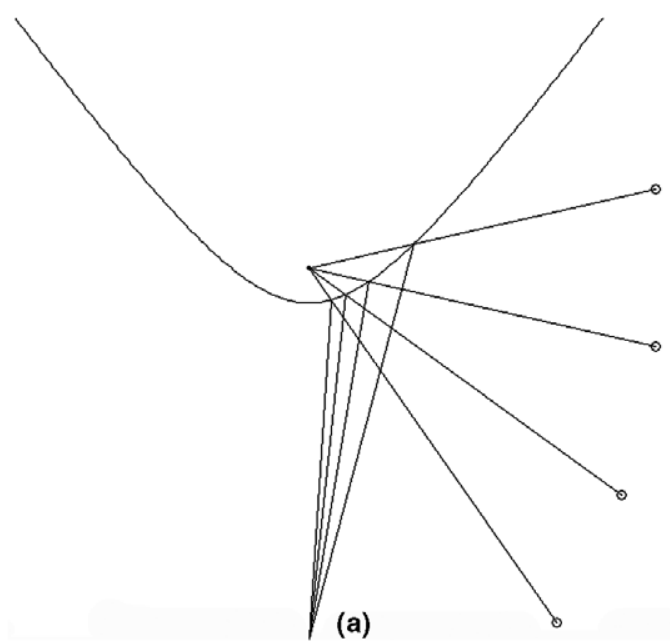

(a)

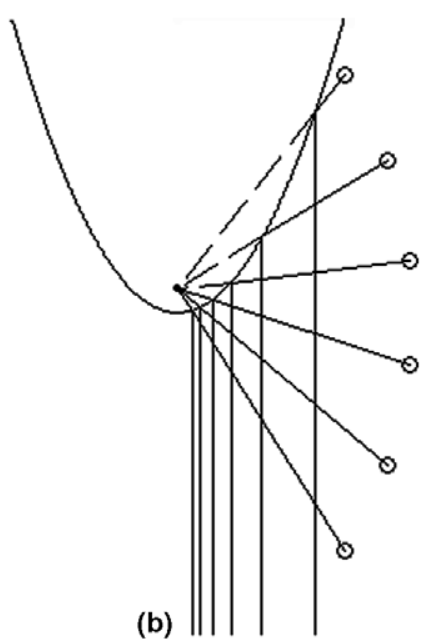

(b)

Fig. 1. SVP catadioptric image formation. (a) Hyperbolic mirror with perspective camera. (b) Parabolic mirror with orthographic camera. 
simultaneous localization and map building with the SYCLOP sensor was presented by Drocourt et al. (2002). Depth perception is recovered by taking into account dead-reckoning information from the rigid translation of the sensor during the navigation. In the special situations when a $3 \mathrm{D}$ scene model is available, the panoramic image can be mapped onto the model to obtain a virtual 3D environment. Researchers from the University of Alberta (Southwell et al., 1996b) used a similar method for pipe inspection using a catadioptric camera with a conical mirror. The omnidirectional image provided by the catadioptric camera is mapped onto a 3D surface defined by a previously available model of the real surface or onto a complex developable surface formed by basic building blocks.

Stereoscopic vision combines separate images taken from distinct points of view and permits depth to be perceived visually. Stereo catadioptric sensors are special structures of mirrors and lenses designed to obtain depth from images with a wide field of view. In order to obtain distinct points of view of a scene with a single camera, several researchers used structures of convex mirrors (Southwell et al., 1996a; Fiala and Basu, 2002; Nene and Nayar, 1998) or planar mirrors (Gluckman and Nayar, 1999, 2002; Kawanishi et al., 1998). For instance, Southwell et al. (1996a) used a non-SVP catadioptric sensor provided with a two lobbed spherical mirror to obtain two images with separated view points that allow panoramic $3 \mathrm{D}$ perception. Later, Fiala and Basu (2002) used a similar sensor to obtain a stereo panoramic view in which horizontal and vertical lines were detected using the Panoramic Hough Transform, an extension of the Hough Transform adapted for catadioptric images. Depth is retrieved by matching the lines imaged by the two spherical mirrors through the radial symmetry. Kawanishi et al. (1998) developed a high-resolution SVP omnidirectional sensor built with six cameras and a hexagonal pyramidal mirror. Stereo views are obtained by connecting two such sensors placed in a back-to-back configuration. Gluckman and Nayar (1999) obtained stereo panoramic views using two planar mirrors and a perspective camera. The sensor produces a stereo image but the whole $360^{\circ}$ image is not built in a single shot which means that a part of the scene is always hidden. Lin and Bajcsy (2003) described an omnidirectional sensor that provides depth information by using two cameras, a beam splitter and a conical mirror. The sensor produces a high-resolution panoramic stereo image but has a small vertical FOV due to the superposition of the view fields of the near camera, the far camera and the conical mirror.

The results obtained from stereoscopic vision are directly related to the accuracy of matching the points between the observed images. The matching problem is actually a difficulty shared by all of the above mentioned panoramic stereo methods.

Structured light-based techniques are a particular case of stereo vision used to solve the correspondence problem in which one of the cameras is replaced by a pattern projector (Salvi et al., 1998, 2003). The use of this technique is similar to placing visible landmarks in the scene so that image points can be identified and matched faster and more accurately. This paper presents an omnidirectional sensor that provides 3D information using structured light. The sensor is formed by a single-camera catadioptric configuration with an embedded omnidirectional structured light projector. The sensor design and the calibration of the whole system is detailed in Section 2. The experimental results and the accuracy measurements are shown in Section 3. The article ends with conclusions and further work.

\section{Sensor geometry and calibration}

As explained in the introductory section, the main goal of the proposed sensor is to retrieve $3 \mathrm{D}$ information from an omnidirectional image to overcome the matching problem present in stereo vision. In the proposed solution, shown in Fig. 2, the omnidirectional camera is coupled with a structured light projector that has a field of view of $360^{\circ}$ (Orghidan et al., 2003). Due to their optical properties, catadioptric sensors having an SVP were chosen to build the omnidirectional camera of the proposed sensor. Commonly, the central projection constraint is fulfilled by the use of a parabolic mirror in conjunction with an orthographic camera or a hyperbolic mirror with a perspective camera. The first configuration, which preserves the SVP independently of the translation between the mirror and the camera, was finally preferred due to its simplicity in focusing the image.

The third dimension of the scene is perceived by crossing the light rays emitted by the laser with the ones observed by the camera or, in other words, performing a triangulation. The laser should project a pattern that covers the whole scene and should be easily identifiable. The chosen pattern was the circle. The light of the projected laser pattern is reflected by the conical mirror and spread onto the scene along an imaginary conical shape. The parabolic mirror reflects the whole scene into the camera and all the spots illuminated by the laser can be immediately identified. With the models for both components of the sensor, a precise triangulation can be carried out. In the following section, we describe the modelling process and the parameter estimation (calibration).

\subsection{Omnidirectional camera calibration}

Assuming that the camera-mirror pair possesses an SVP, the omnidirectional camera can be modelled as a projection onto a sphere followed by a projection onto a plane, as stated by Daniilidis and Geyer (2000), Geyer and Daniilidis (2000). Another way of approaching camera calibration is by considering the mirror surface as a known revolution shape and modelling it explicitly, for instance, considering that the reflecting surface is a paraboloid and the camera is orthographic. Both models were tested and the comparative results were reported in (Orghidan et al., 2005). The omni-camera used for this work has an SVP 


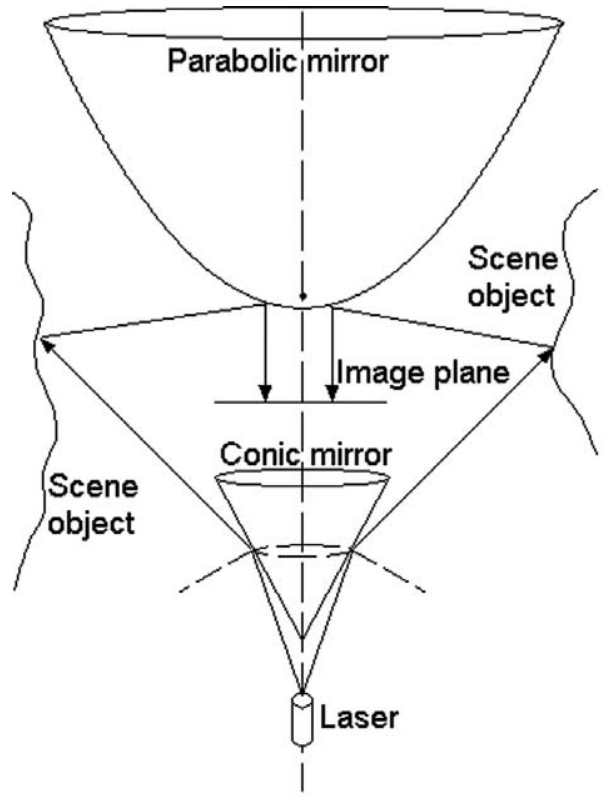

(a)

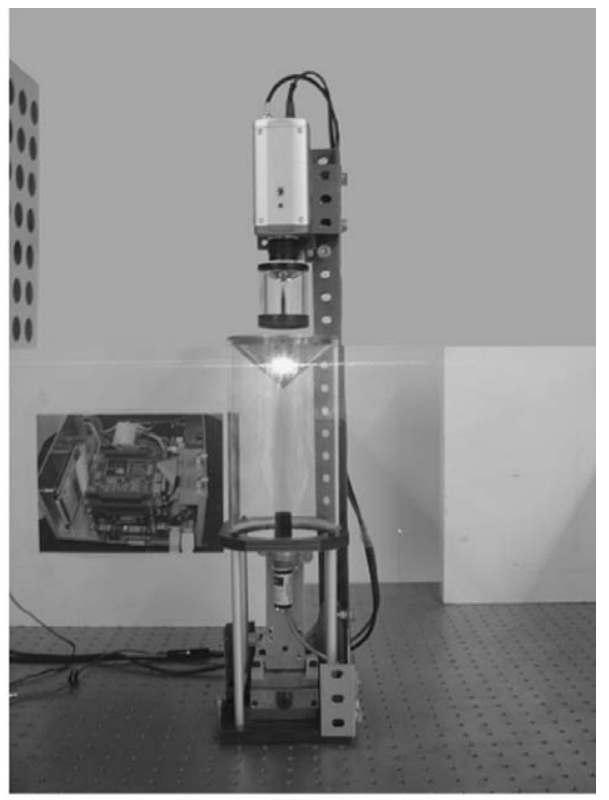

(b)

Fig. 2. The proposed sensor. (a) Catadioptric omnidirectional camera with an embedded structured light projector. (b) Laboratory prototype.

but contains two reflecting surfaces so the first method mentioned (shape independent) was preferred.

The calibration is performed using a set of known 3D points, $P_{w}=\left[x_{w}, y_{w}, z_{w}\right]$, distributed on the four walls of a cube placed around the sensor. Considering $P_{s}=\left[x_{s}, y_{s}, z_{s}\right]$ to be the intersection of the light ray emitted by the point $P_{w}$ with the sphere of radius $R$, then the perspective projection of $P_{s}$ on the image plane from a point $C=[0, \xi]$ produces a point $P_{i}=[x, y]$ as shown in Fig. 3 .

The relation between $P_{w}$ and $P_{s}$ is expressed in Eq. (1).

$\left\{\begin{array}{l}x_{s}=\lambda \cdot x_{w} \\ y_{s}=\lambda \cdot y_{w} \\ z_{s}=\lambda \cdot z_{w}\end{array}\right.$

Since the points belong to the sphere, Eq. (2) also holds

$x_{s}^{2}+y_{s}^{2}+z_{s}^{2}=R^{2}$
The perspective projection of $P_{s}$ on the image plane from a point $C=[0, \xi]$ produces a point $P_{i}=[x, y]$ as expressed in Eq. (3)

$$
\left\{\begin{array}{l}
\frac{x_{s}}{\xi-z_{s}}=\frac{x}{\xi+\varphi} \\
\frac{y_{s}}{\xi-z_{s}}=\frac{y}{\xi+\varphi}
\end{array}\right.
$$

Adding the intrinsic camera parameters $\left(\alpha_{u}, \alpha_{v}, u_{0}, v_{0}\right)$ the pixel coordinates of the image points are shown in Eq. (4)

$$
\left\{\begin{array}{l}
u=\frac{\alpha_{u}(\xi+\varphi) x_{w}}{\xi \sqrt{x_{w}^{2}+y_{w}^{2}+z_{w}^{2}-z_{w}}}+u_{0} \\
v=\frac{\alpha_{v}(\xi+\varphi) y_{w}}{\xi \sqrt{x_{w}^{2}+y_{w}^{2}+z_{w}^{2}-z_{w}}}+v_{0}
\end{array}\right.
$$

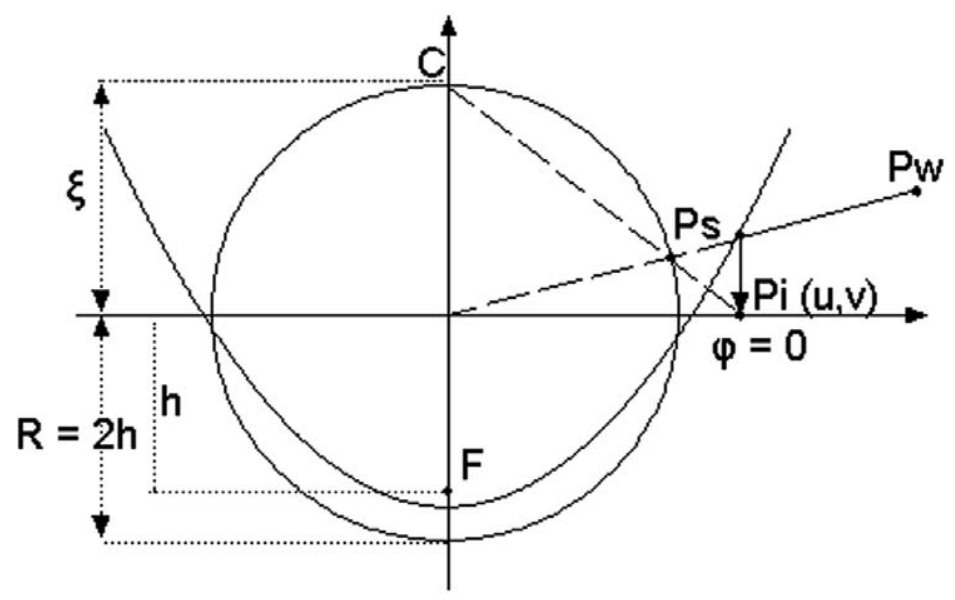

Fig. 3. Image formation using the projective equivalence of an SVP catadioptric projection with the projection on the sphere. 
The parameters of the model are: $\xi$, which depends on the eccentricity; $\varphi$, which is a function of both the eccentricity and the scale; the intrinsic camera parameters $\alpha_{u}, \alpha_{v}, u_{0}$, and $v_{0}$; and $r_{X}(\alpha), r_{Y}(\beta), r_{Z}(\gamma)$ and $t_{x}, t_{y}, t_{z}$, the vectors that model respectively the orientation and the translation between the world coordinate system and the camera coordinate system. The orientation vectors are functions of the three angles $(\alpha, \beta, \gamma)$ which define the rotation on each axis and are expressed in radians while the translations are measured in millimetres. The three rotation angles and the three translations form the set of extrinsic parameters, as detailed in (Salvi et al., 2002).

The difference between the positions of the calculated image points and the positions of the real image points is the calibration error of the model. Minimizing the above error by means of a nonlinear iterative algorithm such as Levenberg-Marquardt, the model of the omnidirectional camera is calibrated.

\subsection{Omnidirectional laser projector model}

The omnidirectional light projector is formed by a laser that emits a circle and is pointed at a conical mirror so that the projected light covers the entire field of view of the catadioptric camera. The proposed projector can be seen as a reversed omni-camera where the light flows in the opposite direction. So, the projector takes advantage of the attributes revealed by previous studies of catadioptric cameras based on the conical mirror shape. Lin and Bajcsy (2001) pointed out that the conical mirror can be used to build true SVP configurations with the advantage that it preserves image point brightness better than other mirrors since it does not distort the image in longitudinal directions. Yagi (1999) highlighted that the conical mirror on a vertical section behaves like a planar mirror and consequently provides a much better resolution than any other omni-mirror shape. Baker and Nayar (1998) proved that the curved mirrors (such as parabolic, hyperbolic, etc.) increase defocus blur because of their bend. Consequently, the cone turns out to be the ideal mirror shape to be used to build the structured light projector.

The bright spots on the scene are observed by the calibrated omnidirectional camera which possesses a unique centre of projection. This property allows the direction of the light source for each image point to be calculated. Since the locations of the calibration planes are known, the 3D coordinates of the laser stripe lying on those planes can be determined. A set of such points can be used to calibrate the laser-conic mirror pair. The 3D projected shape can be represented either as a cloud of points or as a parametric model (Ahn, 2004). The first model mentioned is generally used for object visualization using polygonal mesh surfaces generated from the point cloud. The resulting models describe the object with limited accuracy and require a large amount of computing time and memory space as well. The alternative to the point cloud is the parametric model which provides useful information such as size, shape, posi- tion and rotation of the object. Moreover, the parametric model describes the object in a compact form and needs few points for the parameter estimation. For these reasons, the parametric model was selected to represent the projected 3D shape. The main problems of the parametric model are the analysis and the computational difficulties at the calibration stage when the model is fitted to the experimental data. However, the choice of a set of constraints and a proper model can overcome these drawbacks as we show in the following paragraphs.

A 3D surface can be mathematically described implicitly as stated in Eq. (5), where $a$ is the vector of parameters and $(x, y, z)$ are the coordinates of points in a $3 \mathrm{D}$ space.

$G(a, x, y, z) \cong z-F(a, x, y)=0$

A general 3D curve can be obtained by intersecting two implicit surfaces. The choice of the two surfaces is not trivial and an inappropriate selection can lead to an unnecessarily large set of model parameters, a situation known as overparametrization. The correct constraints have to be found and applied in order to remove the redundant degrees of freedom.

Ideally, when the laser is perfectly aligned with the conic mirror, the $3 \mathrm{D}$ shape formed by the reflected laser pattern can be imagined as a circular cone, called the "laser cone of light". Unfortunately, the precision with which the coordinates of the bright spots are obtained is limited by the catadioptric camera calibration accuracy and by its resolution. Moreover, a perfect alignment of the laser and the conic mirror is difficult to guarantee, so a more general shape than the circular cone should be considered. Since the perspective projection of a circle placed on a plane $\Pi$ onto a plane that is not parallel to $\Pi$ is an ellipse, it can be deduced that a suitable shape for modelling the laser-cone is a revolution surface whose intersection with the plane perpendicular to the omnidirectional camera optical axis is an ellipse. This shape, the elliptic cone, was used in (Orghidan et al., 2005) and proves to be more accurate than the circular cone. Still, for a large amount of noise, the elliptical cone cannot be uniquely determined.

Therefore, the general quadratic surface was chosen to model the laser projection. Let us assume, without loss of generality, that the world reference system is placed such that the calibration planes are perpendicular to the $X$ and the $Y$ axes. The intersections of the quadratic with the calibration planes are arcs described by a subinterval of the parameter domain: the arcs contained in the planes perpendicular to the $X$ and $Y$ axes provide information on the parameters of the quadratic with $x=c t$ and $y=c t$, respectively. Writing the quadratic as in Eq. (6), its intersection with planes $X$ and $Y$ are shown in Eqs. (7) and (8), respectively. The parameters of the arcs for each plane are obtained by fitting the corresponding points into the subsequent equations. Taking into account that the $3 \times 3$ matrix is symmetric, most of the parameters of the quadratic surface can be retrieved from Eqs. (7) and (8). The parameters 
$a_{12}$ and $a_{21}$ correspond to the ellipse obtained by crossing the quadratic with the plane $X Y$.

$$
\begin{aligned}
& {\left[\begin{array}{lll}
x & y & z
\end{array}\right] \cdot\left[\begin{array}{lll}
a_{11} & a_{12} & a_{13} \\
a_{21} & a_{22} & a_{23} \\
a_{31} & a_{32} & a_{33}
\end{array}\right] \cdot\left[\begin{array}{c}
x \\
y \\
z
\end{array}\right]} \\
& +\left[\begin{array}{lll}
x & y & z
\end{array}\right] \cdot\left[\begin{array}{l}
\beta_{1} \\
\beta_{2} \\
\beta_{3}
\end{array}\right]+f=0 \\
& {\left[\begin{array}{ll}
y & z
\end{array}\right] \cdot\left[\begin{array}{ll}
a_{22} & a_{23} \\
a_{32} & a_{33}
\end{array}\right] \cdot\left[\begin{array}{l}
y \\
z
\end{array}\right]+\left[\begin{array}{ll}
y & z
\end{array}\right] \cdot\left[\begin{array}{l}
P_{x} \\
Q_{x}
\end{array}\right]+R_{x}=0} \\
& {\left[\begin{array}{ll}
x & z
\end{array}\right] \cdot\left[\begin{array}{ll}
a_{11} & a_{13} \\
a_{31} & a_{33}
\end{array}\right] \cdot\left[\begin{array}{l}
x \\
z
\end{array}\right]+\left[\begin{array}{ll}
x & z
\end{array}\right] \cdot\left[\begin{array}{l}
P_{y} \\
Q_{y}
\end{array}\right]+R_{y}=0}
\end{aligned}
$$

With the added constraints, the number of parameters to be simultaneously minimized decreases, leading to a more accurate computation.

\section{Experimental results}

The experiments presented in this section are aimed at finding out the reliability of the sensor by evaluating its accuracy measuring depth in a real scene. The section starts with an overview of the sensor calibration. Depth measurements are performed using a laboratory setup and the results are presented and commented on the second part of this section.
The system was built using off the shelf components. The optics and the mirror used for the omnidirectional camera were provided by Remote Reality. The camera is a Sony SSC-DC198P with a $1 / 3$ in. CCD and a resolution of $768 \times 576$ pixels. The laser and its optics are produced by Lasiris, the diode power is $3 \mathrm{~mW}$ and produces red light with a wavelength of $635 \mathrm{~nm}$.

\subsection{Omnidirectional camera calibration}

The camera calibration is performed using points distributed on four planes, placed around the camera at different distances and orientations. The points are the corners of the squares in a checkered pattern. The distance between two adjacent points on the same plane is $5 \mathrm{~cm}$. Four samples of the images used for calibration are presented in Fig. 4. Note that the choice of the calibration pattern is crucial to obtaining good reference points. During our experiments we tested the calibration using both dotted and a checkered patterns. As expected, the calibration using a checkered pattern proved to be more accurate than the one performed using a dotted pattern because of the deformation of the dots after the reflection in the mirror.

The calibrated parameters of the camera-model are listed in Table 1.

The $2 \mathrm{D}$ image error and the $3 \mathrm{D}$ scene reconstruction error have been computed for a sample of 1320 calibration points located on planes placed around the sensor in a range going from $40 \mathrm{~cm}$ up to $80 \mathrm{~cm}$. The average $2 \mathrm{D}$ cal-
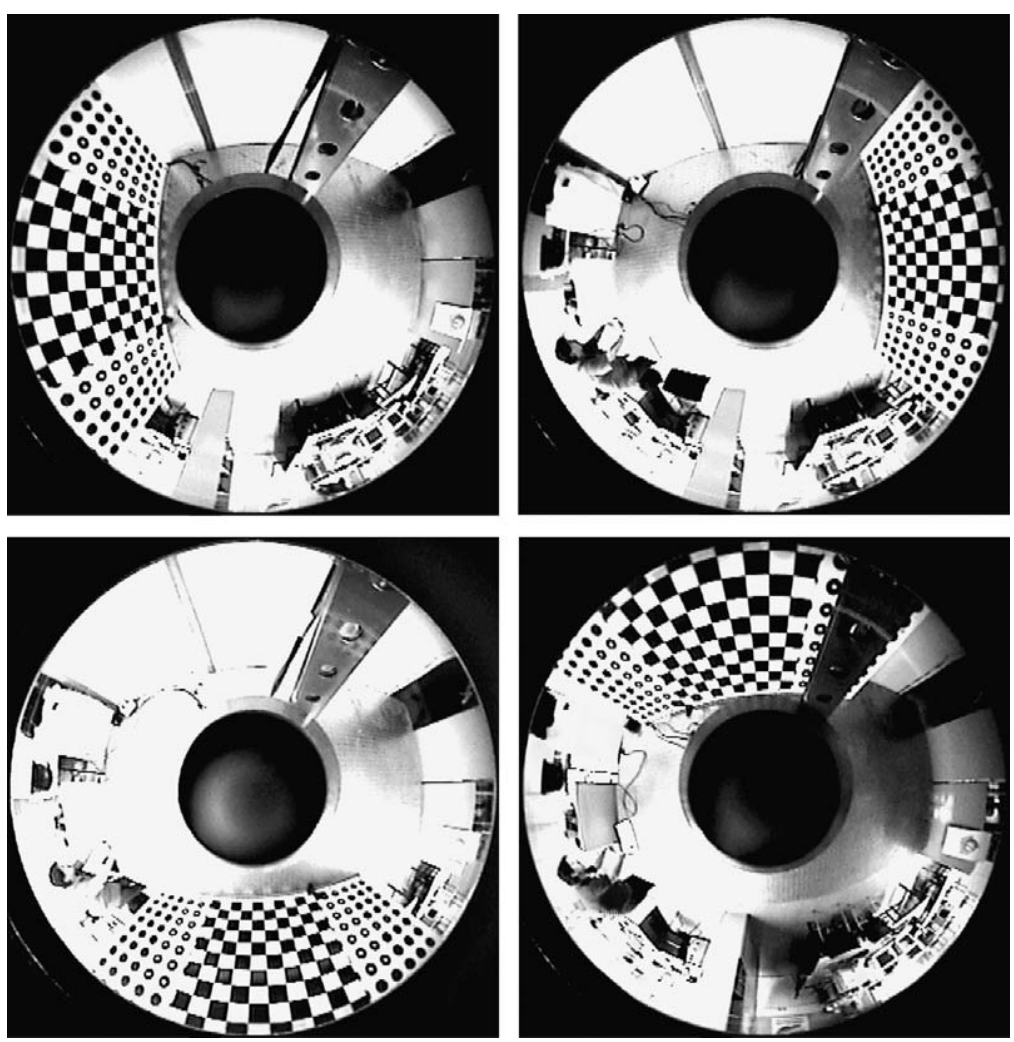

Fig. 4. The calibration planes placed around the omnidirectional camera. 
Table 1

The calibrated parameters for the omnidirectional camera

\begin{tabular}{|c|c|c|c|c|c|c|c|c|c|c|c|}
\hline$\xi$ & $\varphi$ & $\alpha_{u}$ & $\alpha_{v}$ & $u_{0}$ & $v_{0}$ & $\alpha$ & $\beta$ & $\gamma$ & $t_{x}$ & $t_{y}$ & $t_{z}$ \\
\hline 1.07 & -6.12 & -55.10 & 56.84 & 394.23 & 292.20 & -3.08 & -3.12 & -3.13 & 10.22 & 20.94 & -444.29 \\
\hline
\end{tabular}

The measurement units are: the mirror parameters $(\xi, \varphi)$ in $\mathrm{mm}$; the intrinsic parameters $\left(\alpha_{u}, \alpha_{v}, u_{0}, v_{0}\right)$ in pixels; the rotation angles $(\alpha, \beta, \gamma)$ in radians and the translations $\left(t_{x}, t_{y}, t_{z}\right)$ in $\mathrm{mm}$.

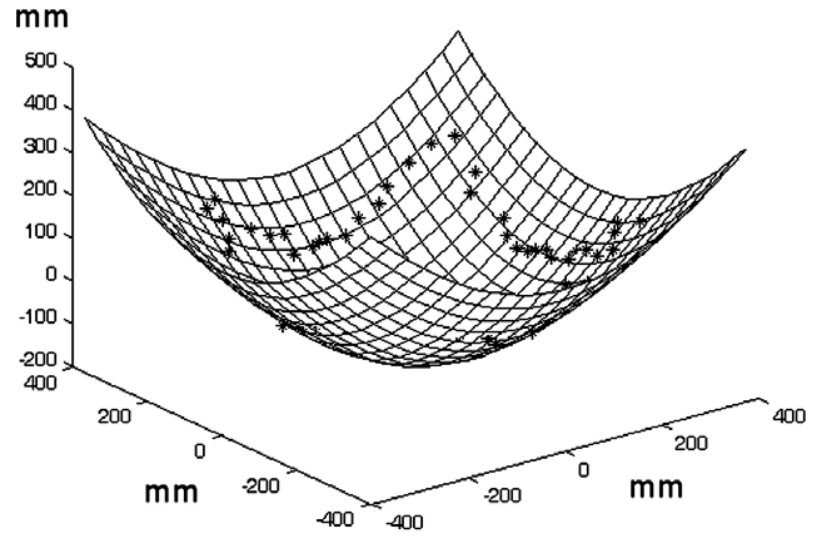

(a)

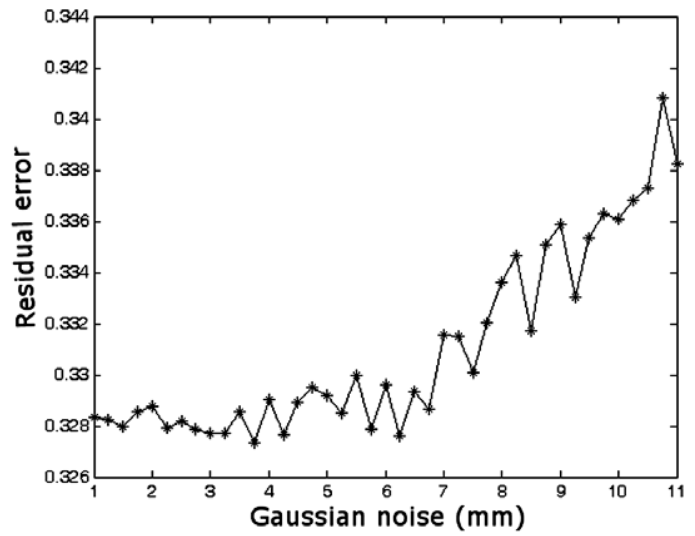

(b)

Fig. 5. Omnidirectional structured light projector calibration. (a) Calibrated quadratic fitted to the 3D points. In the illustrated case the points were affected by a Gaussian noise with $\sigma=5$. (b) The evolution of the error as a function of noise.

ibration error, computed as the distance in pixels from the imaged point and the modelled image of the same point, is $\mu_{2 \mathrm{D}}=1.22$ pixels and the sample standard deviation $\sigma_{2 \mathrm{D}}=1.48$ pixels. The $3 \mathrm{D}$ calibration error was calculated as the distance between the backprojection of the image points on the calibration planes and the $3 \mathrm{D}$ coordinates of the corresponding real points from the calibration pattern. The average 3D calibration error is $\mu_{3 \mathrm{D}}=3.09 \mathrm{~mm}$ and the sample standard deviation $\sigma_{3 \mathrm{D}}=2.75 \mathrm{~mm}$.

\subsection{Omnidirectional laser projector calibration}

The robustness of the calibration method against noise was tested under simulation. We generated a set of 61 points representing the intersection of a cone with the walls of a calibration box having the side-length equal to $50 \mathrm{~cm}$. Then, the three coordinates of the points were perturbed with Gaussian noise having the variance of $\sigma$. The general quadratic shape, representing the projection of the circular laser pattern onto the conic mirror, was fitted to the $3 \mathrm{D}$ points and the resulting surface is shown in Fig. 5(a). The error is considered to be the residual value obtained by plugging the $3 \mathrm{D}$ points into the quadratic equation. Consequently, the residual value for the points lying on the surface equals zero and it increases for points placed away from the surface. The calibration was validated one hundred times for each value of $\sigma$ in the interval [1..11] with a step of 0.25 . The average of the absolute values of the residual errors was calculated at each step. The evolution of the error as a function of noise is presented in Fig. 5(b).
The height of the conic mirror used to build the laboratory prototype is $h=4.4 \mathrm{~cm}$ and the cone aperture angle is $\beta=52^{\circ}$. The laser projects a circular cone with a fan angle $\alpha=11.4^{\circ}$. Since the relation between the two angles is $\beta \approx 0.5(\alpha+\pi / 2)$ the laser is reflected along a very flat surface which can be approximated to a plane: $a x+b y+$ $c z+d=0$, see Fig. 6(b).

The image of the laser is affected by a certain amount of undesired lighting peaks superimposed onto it. This is due to noise produced by four main sources: electrical noise, quantization noise, speckle and image blur. The first two noise sources are associated with the image sensor while the speckle is related to the reduced wavelength of light compared to the surface roughness and the monochromatism of the laser light. Image blur is inherent in the catadioptric cameras due to the mirror curvature. In the omnidirectional image, shown in Fig. 6(a), the center of the laser stripe is determined with sub-pixel accuracy using the peak detection method based on a low pass filter described by Forest et al. (2004). A detail of the laser stripe is shown in Fig. 7(a) in which the effects of the noise on the laser stripe are visible. The detected peaks are represented by red dots in Fig. 7(b) while the green ${ }^{1}$ dots are placed along the ellipse fitted to peaks, plotted with blue.

The intersections between the laser plane and the planes of the calibration box are straight lines which are imaged as elliptical arcs by the omnidirectional camera. Hence, the calibration accuracy is increased by performing an ellipse

\footnotetext{
${ }^{1}$ For interpretation of colour in figures, the reader is referred to the Web version of this article.
} 


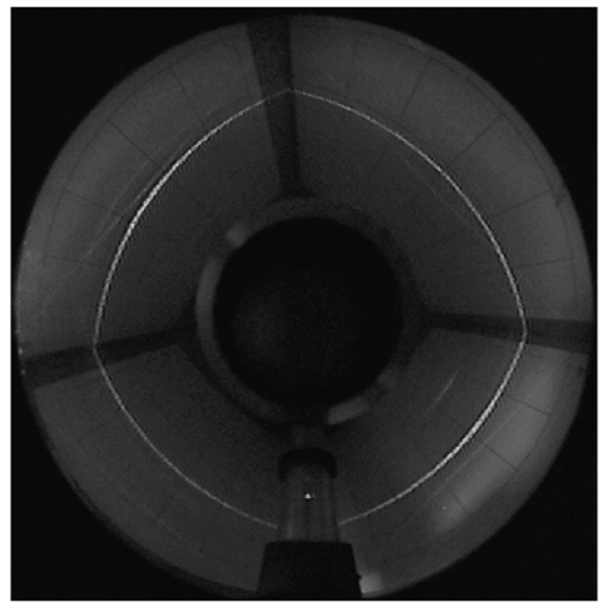

(a)

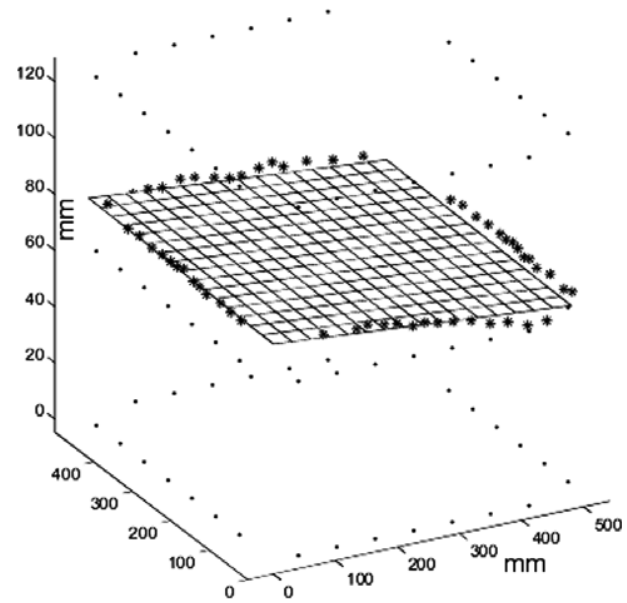

(b)

Fig. 6. Omnidirectional laser projector calibration. (a) Omnidirectional view of the laser pattern. (b) Plane fitted to a set of points of the laser stripe. The dotted rectangles are the points on the planes around the camera.

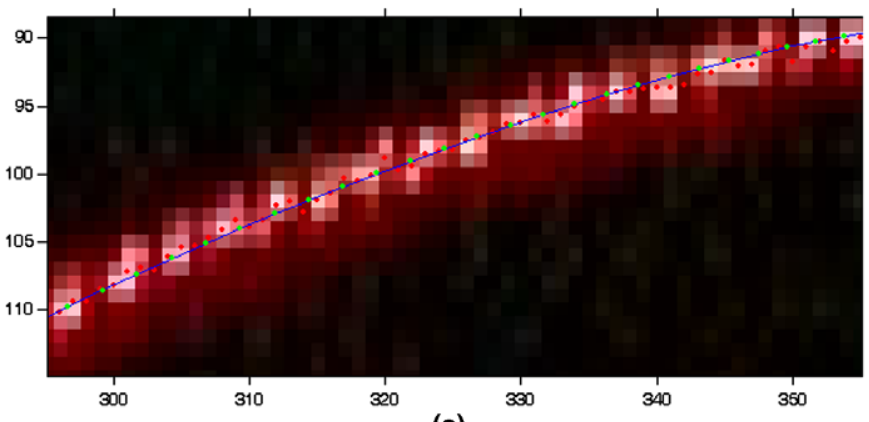

(a)

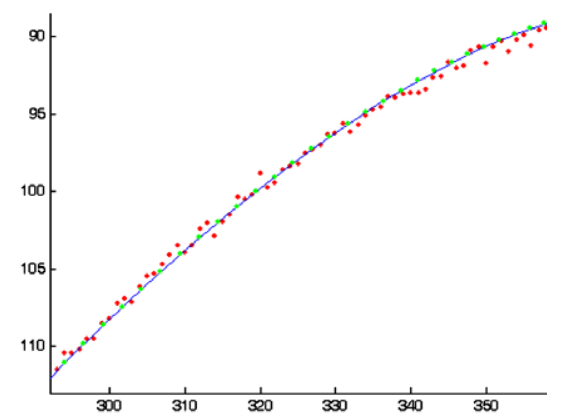

(b)

Fig. 7. Peak detection of a laser stripe in the omnidirectional image. (a) Detail of the real image. (b) Real and approximated points in the omnidirectional image.

fitting for each curve in the image, as shown in Fig. 8. However, this approximation can only be done for those segments of the stripe that represent the projection of the laser pattern onto a plane on the scene.

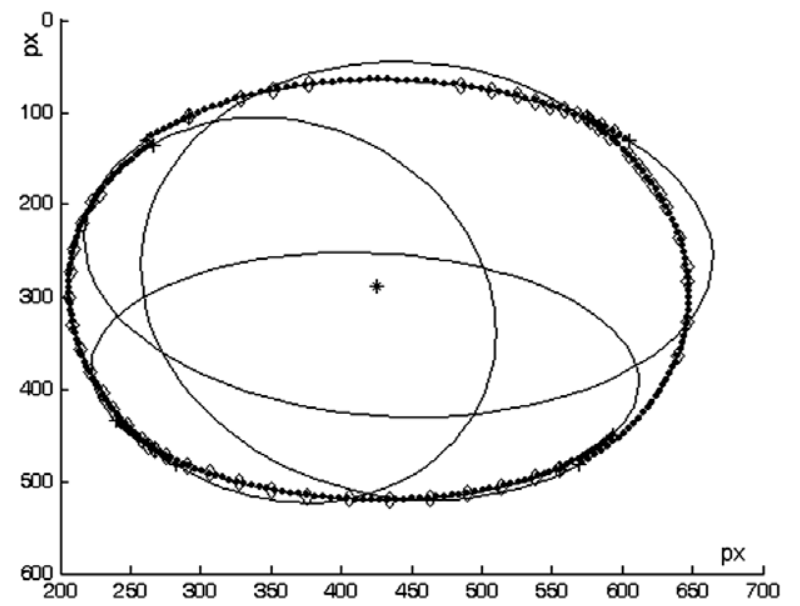

Fig. 8. Ellipse fitting to laser stripes projected on the four calibration walls.

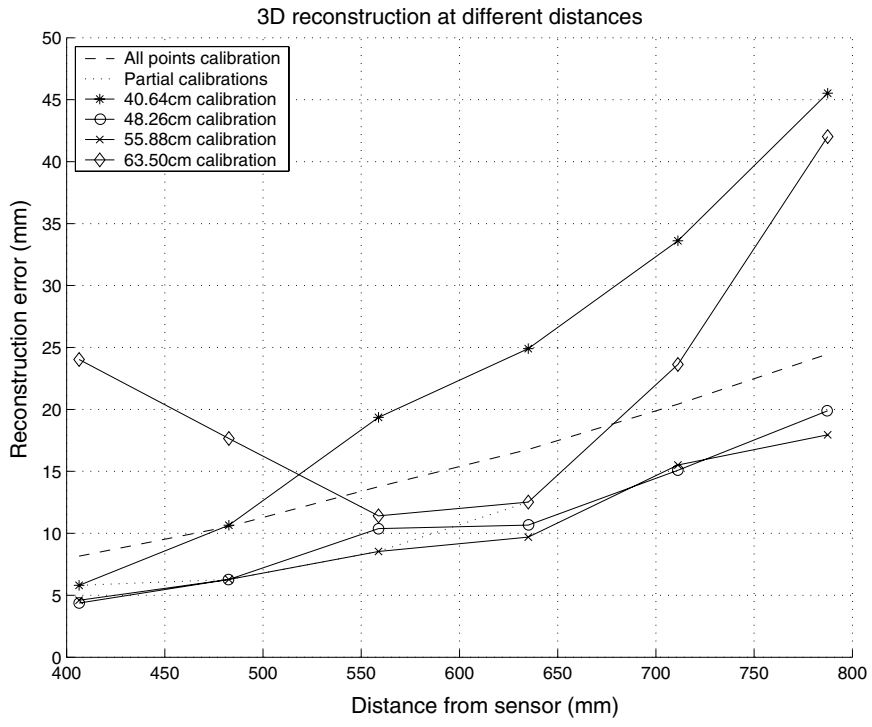

Fig. 9. Accuracy of the proposed sensor with targets placed at different distances and with different model parameters. The dashed line represents the $3 \mathrm{D}$ error with the model obtained by all the calibration points. The dotted line stands for the error using the model obtained when the calibration planes are placed at the same distance as the reference one. The other lines follow the $3 \mathrm{D}$ reconstruction errors of the model with the parameters obtained by the calibrations using planes at different distances. 
The 3D positions of the laser points are calculated using the reversed camera model for the back-projection of the image points on the known calibration planes. For a more accurate calibration, the laser plane was fitted to a cloud of points obtained by the back-projection of the laser image points on several calibration planes placed at different distances and orientations with respect to the sensor.

\subsection{Scene reconstruction and accuracy estimation}

As in traditional stereo, the proposed sensor allows depth perception when its two components are able to generate disparity up to a certain amount. The disparity, which is the angular difference between two different viewing directions of the same point, is directly related to the depth of the observed point and the distance between the "eyes", known as baseline. Even though our sensor does not deal with two images, its capability to perceive depth within a certain range is strongly connected to the length of its baseline. In the case of the presented sensor the baseline is the distance between the camera view point and the laser plane, along the optical axis. At the design stage, the baseline has been implicitly chosen because the laser stripe had to be visible in the central part of the omnidirectional image. After calibration, the length of the baseline was calculated to be $95.26 \mathrm{~mm}$. Note that, due to the SVP property of the catadioptric camera, the baseline is constant and does not depend on the elevation of the observed scene point, which is not the case for nonSVP stereo catadioptric systems.

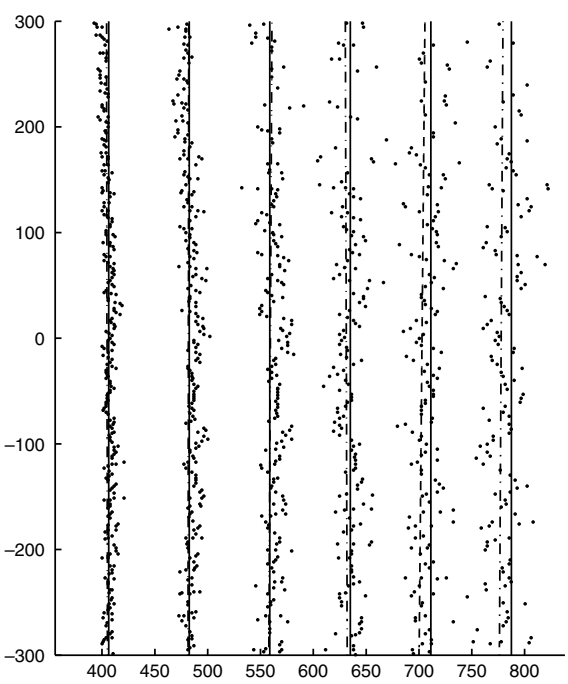

(a)

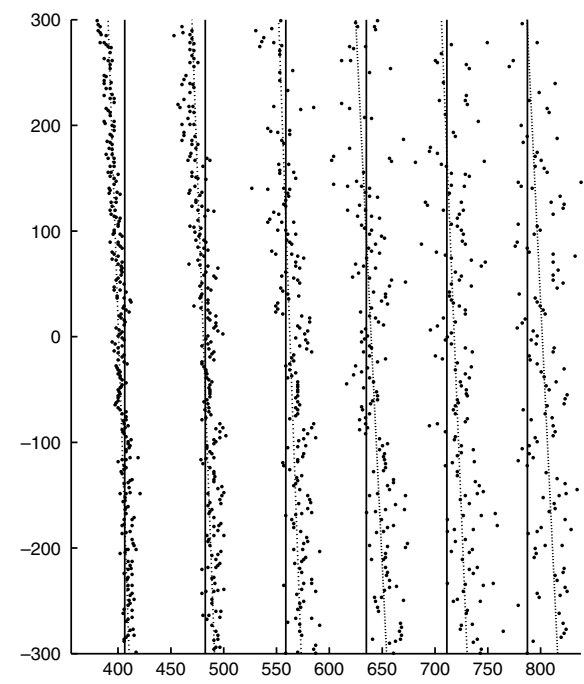

(b)

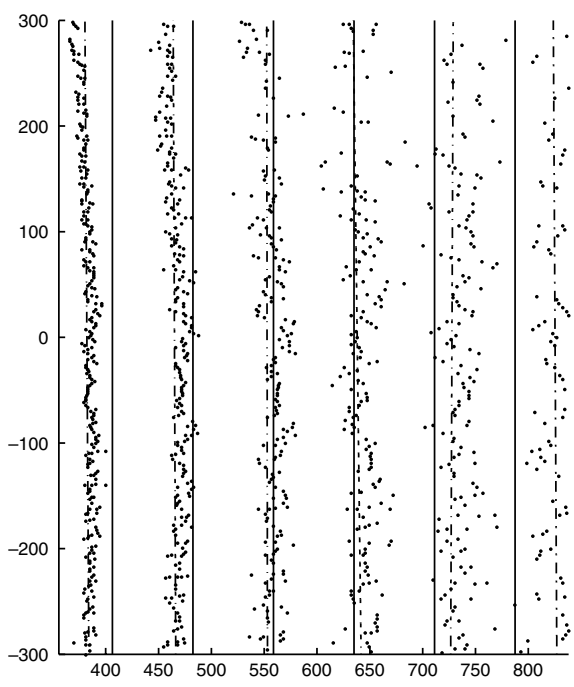

(c)

Fig. 10. Depth perception for planes at different positions. The continuous lines represent the real positions of the planes. The dotted lines are the planes fitted to the estimated points. (a) Calibration using only the calibration planes at $55.88 \mathrm{~cm}$. (b) Calibration using all the points. (c) Calibration using only the calibration planes at $63.50 \mathrm{~cm}$. 
In order to estimate the sensor accuracy, we placed a planar surface at different known positions and its location was estimated. The mean error is the average of the distances of the 3D points reconstructed by the sensor to the reference plane. The plane was placed at different distances from the sensor within a range from $40.62 \mathrm{~cm}$ (16 in.) to $78.74 \mathrm{~cm}(31 \mathrm{in}$.) and the error was calculated for each position. Note that the sensor calibration was performed several times using calibration planes placed at different distances from the sensor. This technique is particularly useful for the proposed sensor since the laser stripe shows up in the omnidirectional image at unpredictable distances from the image centre. As shown in Fig. 9, the $3 \mathrm{D}$ error has a smooth variation when the calibration parameters obtained using points taken at different distances are plugged into the model (the dotted line). A higher variation is obtained when the reconstruction is performed using any other set of parameters, obtained by placing the calibration planes at the same distance from the sensor.

The reconstruction of planes placed at different positions and using different model parameters are presented in Fig. 10. The measurable quantities represented on both axes are expressed in millimetres.

\section{Conclusions and future work}

This paper is focused on combining omnidirectional vision and structured light with the aim of obtaining panoramic depth information. The resulting sensor is formed by a single catadioptric camera and an omnidirectional light projector. The two omnidirectional systems that compose the sensor are calibrated and the resulting model is used to measure distances in a real scene. The experimental results show that the shapes are properly retrieved and the average error of the sensor is less than $2.5 \mathrm{~cm}$ for obstacles placed in a range of $80 \mathrm{~cm}$ in any direction. The results obtained are encouraging and prove that this sensor can be used with depth perception industrial applications such as pipe inspection. The sensor is capable of finding obstacles placed vertically, common in man made environments. Moreover, when previous information about the scene geometry is available the accuracy can be increased by using this information at the segmentation stage for removing potential noise sources. The provided depth information is reliable for close target distances making the sensor suitable for industrial inspection from a slow moving platform. The use of $360^{\circ}$ images and scene-depth information is ideal for robot navigation tasks, especially in obstacle avoidance and map building.

The single-camera catadioptric systems benefit from a wide field of view at the expense of a resolution that is lower than common cameras. Therefore, such an omnidirectional device should be used for gathering information from the surrounding scene and is not suitable for wide scenes or for very accurate detail detection. Following this logic, we conclude that the proposed sensor is appropriate for applications that need a large field of view and fairly low accuracy. High accuracy can be obtained if the sensor is used in conjunction with a perspective camera that could provide more precise image details for some parts of the scene. The structured light projection can be used by both the omnidirectional and the perspective cameras for depth calculations.

An inherent disadvantage of the proposed sensor is that it can recover only one line of $3 \mathrm{D}$ dots at one position. The first prototype can be improved by the use of a more complex structured light pattern. For instance, a set of concentric circles or colour coded light patterns can increase the vertical FOV for stereo perception as much as the overlapping between the conical mirror and the parabolic mirror fields of view. Thus, full cylindrical surfaces of $3 \mathrm{D}$ dots could be recovered. However, it is not always necessary or worthwhile to assume the costs of building the entire $3 \mathrm{D}$ cylinder. If only a small region is required for a particular application or the sensor moves in a well structured environment then less computing time is necessary to build that region.

Another shortcoming of the proposed sensor is its range. The laser used for the prototype presented here is not visible at distances greater than $80 \mathrm{~cm}$. A stronger laser would overcome this problem. The only concern about increasing the laser strength is the eye safety restrictions that might apply in the environment where the sensor is used. Moreover, the sensor detection range can be increased by using optical filters adapted to the laser frequency and a higher resolution camera in order to optimize the laser detection.

\section{References}

Ahn, S.J., 2004. Least-squares orthogonal distance fitting. Lecture Notes in Computer Science, vol. 3151.

Baker, S., Nayar, S.K., 1998. A theory of catadioptric image formation. In: IEEE Sixth Internat. Conf. on Computer Vision, pp. 35-42.

Bruckstein, A.M., Richardson, T.J., 2000. Omniview cameras with curved surface mirrors. In: First Workshop of Omnidirectional Vision, pp. 79-84.

Daniilidis, K., Geyer, C., 2000. A unifying theory for central panoramic systems and practical applications. In: IEEE Sixth European Conf. on Computer Vision, pp. 445-461.

Drocourt, C., Delahoche, L., Marhic, B., Clerentin, A., 2002. Simultaneous localization and map construction method using omnidirectional stereoscopic information. In: IEEE Internat. Conf. on Robotics and Automation, vol. 1, pp. 894-899.

Fiala, M., Basu, A., 2002. Panoramic stereo reconstruction using non-SVP optics. In: 16th Internat. Conf. on Pattern Recognition, vol. 4, pp. 27 30 .

Forest, J., Salvi, J., Cabruja, E., Pous, C., 2004. Laser stripe peak detector for 3D scanners, a FIR filter approach. In: Internat. Conf. on Pattern Recognition, vol. 3, pp. 646-649.

Gaspar, J., Decco, C., Okamoto Jr., J., Santos-Victor, J., 2002. Constant resolution omnidirectional cameras. In: Third Workshop of Omnidirectional Vision, pp. 27-34.

Geyer, C., Daniilidis, K., 2000. Equivalence of catadioptric projections and mappings of the sphere. In: First Workshop of Omnidirectional Vision, pp. 91-96. 
Gluckman, J., Nayar, S.K., 1999. Planar catadioptric stereo: Geometry and calibration. In: IEEE Computer Vision and Pattern Recognition, vol. 1 , pp. 22-28.

Gluckman, J., Nayar, S.K., 2002. Rectified catadioptric stereo sensors. IEEE Trans. Pattern Anal. Machine Intell. 24 (2), 224-236.

Hicks, R.A., Perline, R.K., 2001. Geometric distributions for catadioptric sensor design. In: IEEE Computer Society's Computer Vision and Pattern Recognition, vol. 1, pp. 584-589.

Kawanishi, T., Yamazawa, K., Iwasa, H., Takemura, H., Yokoya, N., 1998. Generation of high-resolution stereo panoramic images by omnidirectional imaging sensor using hexagonal pyramidal mirrors. In: Fourteenth Internat. Conf. on Pattern Recognition, vol. 1, pp. 485489.

Lin, S.S., Bajcsy, R., 2001. The true single view point (SVP) configuration for omni-directional view catadioptric system using cone mirror. Technical report ms-cis-00-24, Computer and Information Science Department, University of Pennsylvania, Philadelphia, USA.

Lin, S.S., Bajcsy, R., 2003. High resolution catadioptric omni-directional stereo sensor for robot vision. In: IEEE Internat. Conf. on Robotics and Automation, pp. 1694-1699.

Nene, S.A., Nayar, S.K., 1998. Stereo with mirrors. In: Sixth Internat. Conf. on Computer Vision, pp. 1087-1094.

Orghidan, R., Salvi, J., Mouaddib, E.M., 2003. Calibration of a structured light-based stereo catadioptric sensor. In: Workshop on Omnidirectional Vision, IEEE Conf. on Computer Vision and Pattern Recognition.
Orghidan, R., Mouaddib, E.M., Salvi, J., 2005. Omnidirectional depth computation from a single image. In: IEEE Internat. Conf. on Robotics and Automation, pp. 1234-1239.

Pegard, C., Mouaddib, E.M., 1996. A mobile robot using a panoramic view. In: IEEE Internat. Conf. on Robotics and Automation, vol. 1, pp. 89-94.

Salvi, J., Batlle, J., Mouaddib, E., 1998. A robust-coded pattern projection for dynamic 3D scene measurement. Pattern Recognition Lett. (19), $1055-1065$.

Salvi, J., Armangue, X., Batlle, J., 2002. A comparative review of camera calibrating methods with accuracy evaluation. Pattern Recognition 35 (7), 1617-1635.

Salvi, J., Pagès, J., Batlle, J., 2003. Pattern codification strategies in structured light systems. Pattern Recognition 37 (4), 827-849.

Southwell, D., Basu, A., Fiala, M., Reyda, J., 1996a. Panoramic stereo. In: 13th Internat. Conf. on Pattern Recognition, vol. 1, pp. 378-382.

Southwell, D., Vandegriend, B., Basu, A., 1996b. A conical mirror pipeline inspection system. In: IEEE Internat. Conf. on Robotics and Automation, 22-28 April 1996, vol. 4, pp. 3253-3258.

Svoboda, T., Pajdla, T., 2000. Panoramic cameras for 3D computation. In: Proc. Czeck Pattern Recognition Workshop, pp. 63-70.

Yagi, Y., 1999. Omnidirectional sensing and its applications. In: IEICE Trans. on Information and Systems, vol. E82-D, pp. 568-578.

Yagi, Y., Kawato, S., 1990. Panoramic scene analysis with conic projection. In: IEEE/RSJ Internat. Conf. on Intelligent Robots and Systems, vol. 1, pp. 181-187. 